\title{
Minimally Invasive Percutaneous Fixation of Non-United Scaphoid Fractures
}

\author{
AHMED EL-XENI, M.Sc.; AHMED KHOLEIF, M.D.; SHERIF KHALED, M.D.; MOSTAFA MAHMOUD, M.D.; \\ MOHAMED RAMADAN MOHAMED, M.Sc.; AHMED B. AL-SHAZLI, M.Sc. and \\ ASHRAF YASSIN AMER, M.Sc.
}

The Department of Orthopedic Surgery, Faculty of Medicine, Cairo University

\begin{abstract}
Background: Scaphoid non-unions pose a great challenge to surgeons because of the multiple factors that may contribute to their causation.

The etiology of the nonunion may be because of anatomic variations, fracture configuration, vascular problems, underlying metabolic problems, or the inadequacy of initial treatment.

Percutaneous management of scaphoid non-unions offers the advantage of inducing minimal trauma to the soft tissues while adequately stabilizing the fracture site to induce union in a high percentage of cases.

This study proves that percutaneous fixation of delayed or nonunited scaphoid fractures result in predictable satisfactory union rate and functional outcome, it proves that percutaneous Herbert's screw insertion carries no risk of damage to soft tissues or vascular supply. Also, the gap is not the determinant of time to union but actually it is the time since injury; the longer the time since injury the longer the time to union as long as the scaphoid alignment is maintained i.e. no humpback deformity.
\end{abstract}

Key Words: Nonunion-Scaphoid-Headless screw-Percutaneous.

\section{Introduction}

FRACTURES of the scaphoid are common and affect predominantly young and productive individuals. The highly mobile scaphoid plays a critical role in coordinating the kinematics of the two carpal rows and is subject to considerable compressive, rotational, and shear forces during hand and wrist motion. In addition, the scaphoid has a peculiar and limited retrograde blood supply that is vulnerable to disruption by trauma; consequently, scaphoid fractures are prone to a high rate of

Correspondence to: Dr. Ahmed El-Xeni, The Department of Orthopedic Surgery, Faculty of Medicine, Cairo University delayed union and non-union that may advance to wrist osteoarthritis if left untreated [1].

Open treatment and bone grafting for nonunions has an established track record. The advantage of these techniques is that they allow direct visualization of the fracture fragments for freshening, reduction, and correction of associated deformities, however, an open operative approach is well recognized for some disadvantages, including the need to expose and divide the palmar radiocarpal ligaments, the potential for interruption of the already tenuous blood supply, as well as the possibility of a hypertrophic and painful scar at the wrist crease, so the application of minimally invasive procedures for scaphoid nonunion attempts to combine appropriate reduction and rigid fixation with minimal disruption of the soft tissues $[2,3]$

It is important to understand that minimally invasive techniques are not applicable to all types of scaphoid nonunion; essentially, minimally invasive surgery is indicated for scaphoid non unions without collapse of the scaphoid architecture, and without complete AVN of the proximal pole. These fractures usually have a cartilaginous shell intact externally and have a normal scapho-lunate angle without a humpback deformity allowing the use of cannulated headless screw which is the most popular method of fixation $[3,4]$.

\section{Patients and Methods}

Between 2011 and 2013 in Kasr Al-Ainy Hospitals and El-Agouza Police Hospital, we prospectively studied Thirty one patients with more than 6 months duration of scaphoid fractures that were fixed percutaneously by Herbert screws and followed-up for a period of 8-24 months (average, 
17 months). Patients were 30 men and 1 woman ranging between 19-40 years (average of 28.3 years). With mean time between injury and operation of 17.2 months (6 to 36). The dominant hand was affected in 18 patients and the non-dominant in 13. Fall on outstretched hand (FOOSH) was the most common mode of trauma causing the fracture in 20 patients; motorcycle accident was the second common mode of trauma in 9 patients and during athletic activity was found in two. Twenty four patients had initial management in thumb Spica cast ranged from 4 to 12 weeks, while 7 patients had no initial period of immobilization due to missed diagnosis, none was a smoker. Patients were evaluated for clinical and radiographic results.

The patients presented with a painful wrist that was worse on exertion, and with tenderness in the anatomical snuffbox. Grip strength and range of movement were recorded, and pain was measured by a visual analogue scale (VAS) ranging from 0 (no pain) to 10 (maximum pain). Further evaluation used the Mayo modified wrist score. No patient gave history or presented with associated injury. Plain radiography wrist PA, lateral and scaphoid views were done. Preoperative CT scan was done in all cases to evaluate displacement, deformity, gap size, arthritis or sclerosis, and none had undergone previous wrist surgery. Cases were classified according to Slade \& Dodds classification 14 were grade IV (gap $<5 \mathrm{~mm}$ ) and 16 were grade V (gap $>5 \mathrm{~mm}$ ) and one grade IV subtype A. All patients had waist fractures except one with proximal pole fracture. By Computed tomography 14 patients had gap $<5 \mathrm{~mm}, 16$ patients with gap $>5 \mathrm{~mm}$ and one proximal pole fracture (with gap $<5 \mathrm{~mm}$ ), all patients in the sample presented with sclerosis $<2 \mathrm{~mm}$ on CT, patients with more sclerosis were excluded from the study inspected by 2 independent radiologists. All patients showed no humpback deformities or carpal malalignment denoting neither ligamentous injury nor AVN on CT. By Magnetic resonance imaging (MRI)there were no ligamentous injuries or evidence of avascular necrosis for 12 patients provided MRI study, and no patient presented with AVN or ligamentous injury latter on during follow-up. No associated injury detected by neither past history nor examination in the sample.

The inclusion criteria were a nonunion of a fracture of the scaphoid of at least six months' duration, with bone resorption of $>2 \mathrm{~mm}$, and the absence of humpback deformity, excessive sclerosis or arthritis. Humpback deformity was excluded as it is a sign of erosion of the volar cortex which requires bone grafting for correction. 29 patients fixed through volar approach and 2 patients through dorsal approach. The point of entry of the wire in volar approach was actually through the trapezial ridge. Also in cases with sclerosis we used multiple wires for drilling through sclerosis to refresh fracture edges. A wire of the same length is used to measure screw length, after which guide wire is advanced to radius to prevent wire pullout during drilling. In the dorsal approach blunt dissection to the bone through the snip wound was done to free out extensor tendons before drilling and screw application to make sure that they are not tethered during the procedure. A short arm cast excluding the thumb was worn for six weeks, followed by a removable splint for four weeks. Patients were allowed to exercise the wrist after removal of the cast, but instructed to avoid contact sports or full loading until union was achieved.

Radiological examination at 4 weeks and every 2 weeks until union was achieved for assessment of screw position, carpal alignment and evidence of complications; arthritis or nonunion then at 6 months and after one year for follow-up. Serial clinical examination at time of union, 6 months and at the last follow-up for evaluation of the functional outcome using Mayo modified wrist score and patient related wrist evaluation (PRWE).

\section{Results}

No patients were lost at follow-up, all patients had tenderness on the anatomical snuffbox and the scaphoid tubercle that gets worse with exertion. 13 patients had tenderness on first metacarpal axial loading. 19 patients reported anatomical snuff box swelling after exertion.

Tenderness over the lunate, capitate or Scapholunate joint could not be differentiated from the fracture pain. 28 patients reported that this pain was affecting their jobs or daily lives. There was no sign of nerve entrapment or compartment syndrome. There was no muscle wasting or scars of previous operations at both.

Twenty four patients were subjected to paid sick leaves. Postoperatively there was less pain, either in the snuffbox or with wrist movement, and the VAS (visual analogue scale) improved from a mean of 4.1 ( 3 to 6 ) to a mean of 1.07 ( 0 to 2 ). The mean pre-operative range of movement was $43.2^{\circ}\left(30^{\circ}\right.$ to $\left.60^{\circ}\right), 50.6^{\circ}\left(35^{\circ}\right.$ to $\left.70^{\circ}\right), 5.8^{\circ}\left(0^{\circ}\right.$ to $\left.15^{\circ}\right)$ and $15.4^{\circ}\left(10^{\circ}\right.$ to $\left.25^{\circ}\right)$ for extension, flexion, radial deviation and ulnar deviation, respectively. The postoperative range of movement improved to means of $61.6^{\circ}\left(45^{\circ}\right.$ to $\left.80^{\circ}\right), 65^{\circ}\left(55^{\circ}\right.$ to $\left.80^{\circ}\right)$, 
$10.8^{\circ}\left(5^{\circ}\right.$ to $\left.20^{\circ}\right)$ and $26.1^{\circ}\left(20^{\circ}\right.$ to $\left.35^{\circ}\right)$ for extension, flexion, radial deviation and ulnar deviation, respectively. Pre-operatively, the Mayo modified wrist score was poor in 18 patients $(58.06 \%)$, fair in $12(38.7 \%)$ and good in one $(3.2 \%)$. This im- proved to 28 excellent $(90.3 \%)$ and three good (9.6\%). Preoperatively PRWE mean was 36.6 (20$60)$, improved to mean of 6.45 (0-20). Radiologically all fractures healed in a mean time 11.6 weeks ranging from (8-16 weeks).

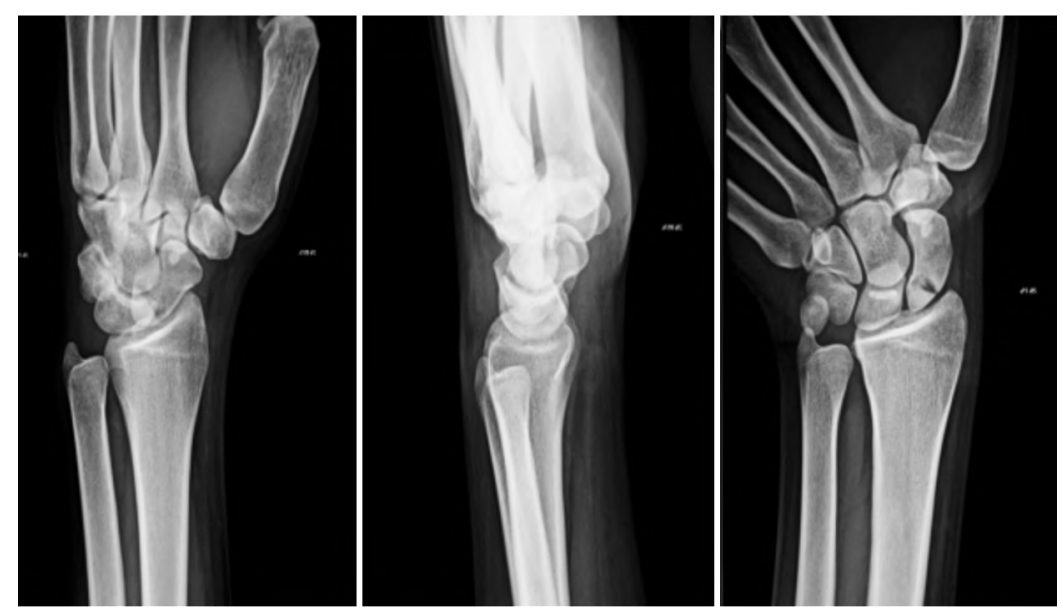

Fig. (1A): Pre-operative X-rays of a 19 years old patient with missed fracture scaphoid since 12 months.
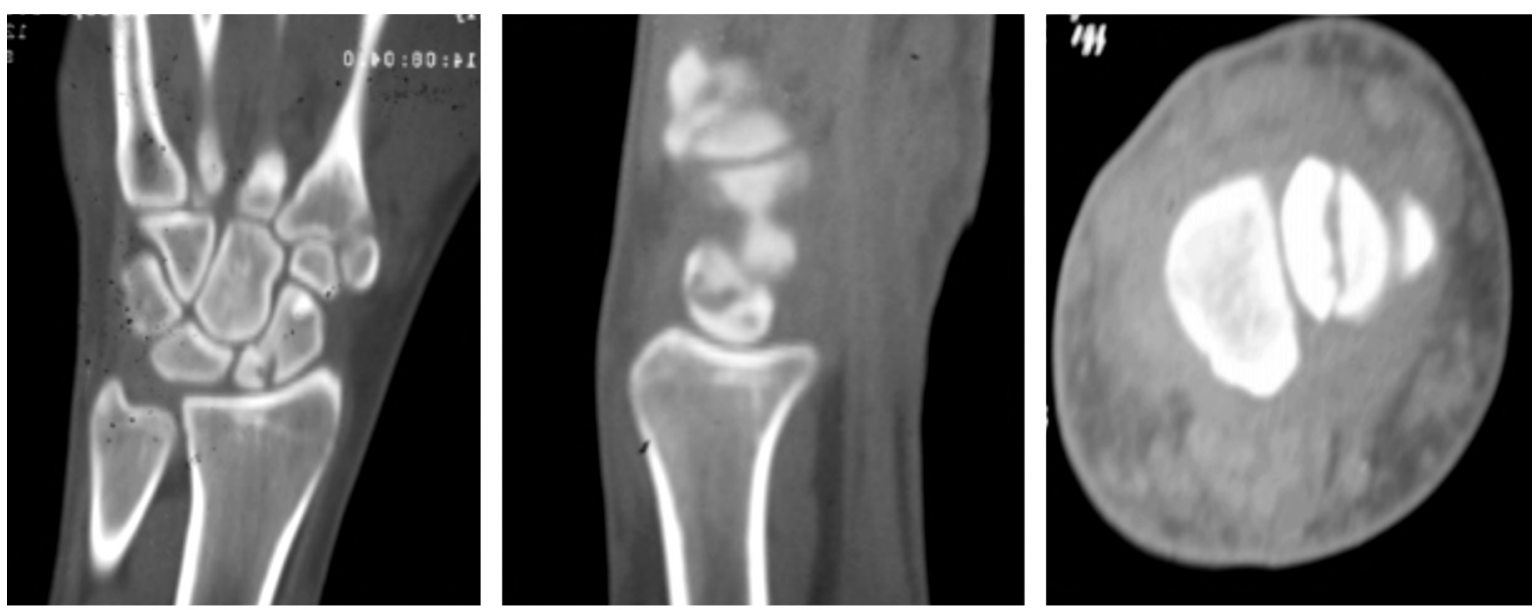

Fig. (1B): CT of a 19 years old patient with missed fracture scaphoid since 12 months.
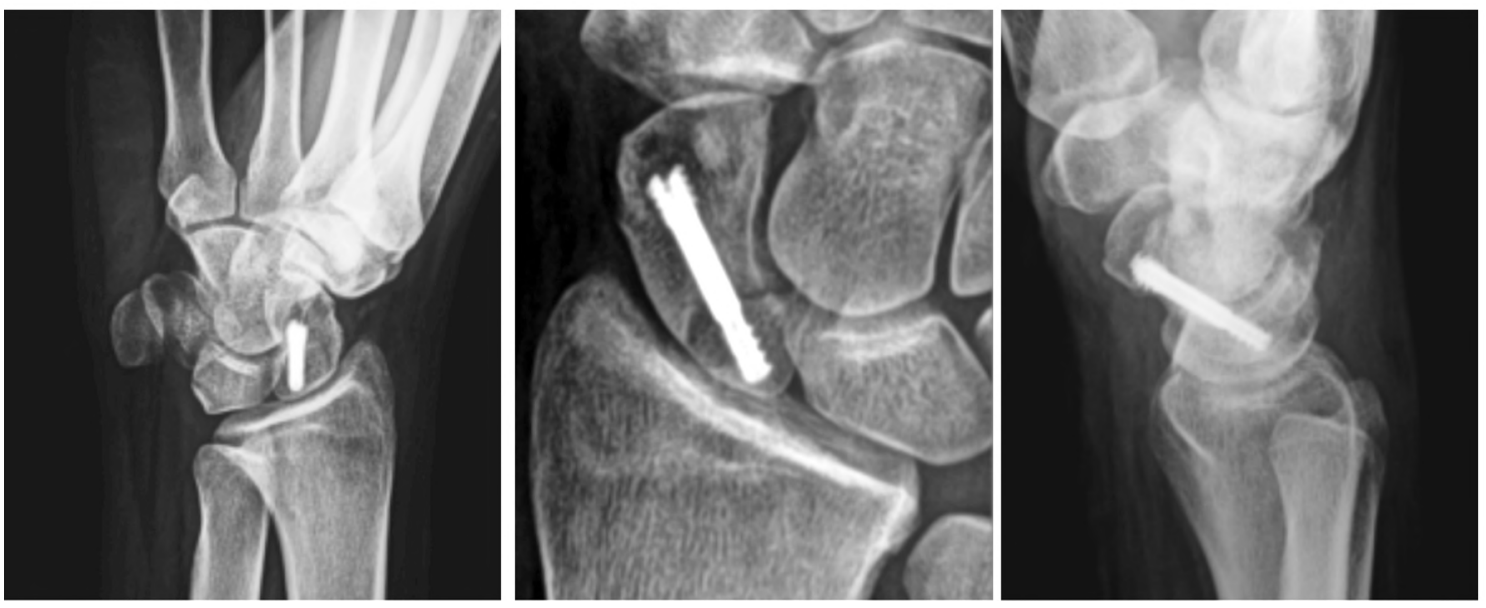

Fig. (2A): Eight months post-operative X-rays. 

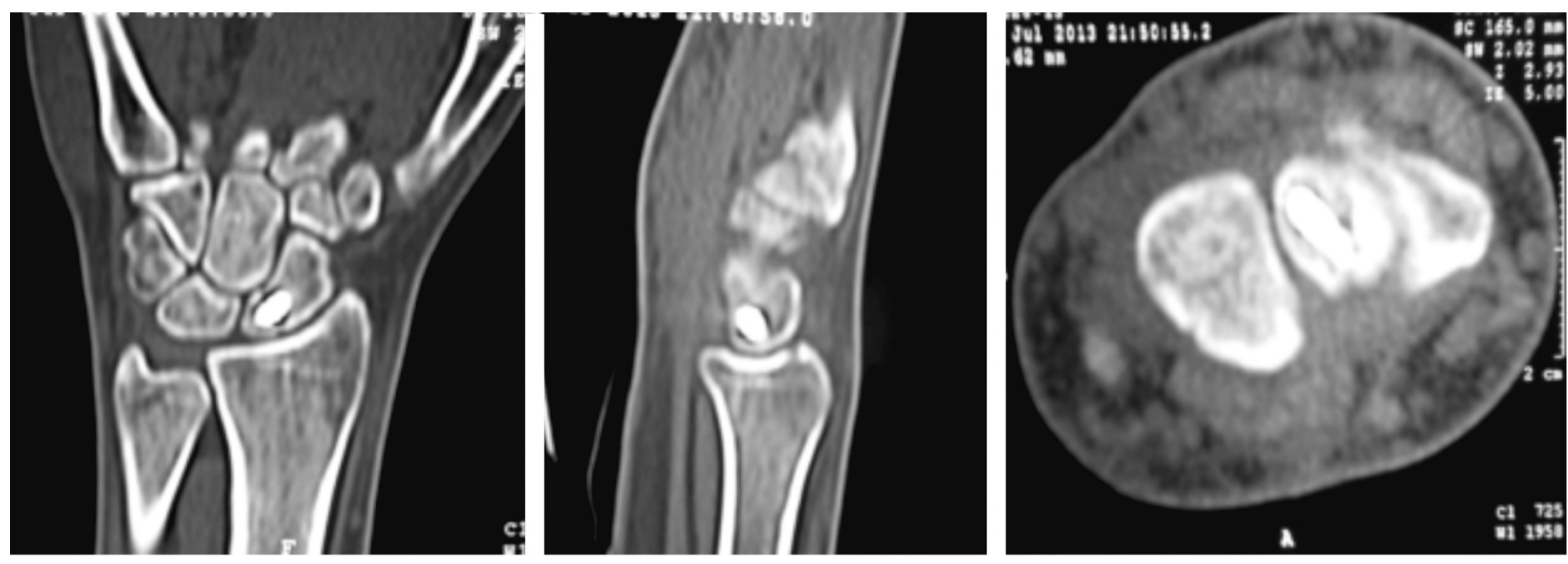

Fig. (2B): \& CT showing union.

\section{Discussion}

The scaphoid, like the talus or head of femur, has an almost complete cartilaginous surface except for small portions on the dorsal and palmar aspect. Consequently, it depends on an intra-osseous healing process especially in the proximal one-third that is supplied from intra-osseous vessels. Therefore, there is greater possibility of a prolonged healing time and non-union in the more proximal fracture [5]. An important milestone in the surgical treatment of scaphoid fractures was the introduction of the Herbert screw. The introduction of cannulated screws has made it possible to fix the scaphoid fractures using a minimal invasive technique. The damage to the palmar carpal ligaments could be avoided and the scar would be much better. Fibrous union of scaphoid fractures occurs because the healing process is arrested in its early stages due to micro-movement and lack of mechanical stabilization [6]. Barton [7] studied a group of such fibrous unions that had initially appeared to be solidly healed. However, there had been insufficient remodeling to resist bending and torque, and half of these fractures subsequently developed nonunion.

Non-unions with minimal sclerosis are similar to fibrous unions and require only rigid fixation for healing. Healing can still proceed in a stable fracture with a gap < $1 \mathrm{~mm}$, [8] and Cosio \& Camp [9] stabilized such non-unions with multiple Kirschner wires, achieving solid union in $77 \%$ of their cases without open repair or bone grafting. During open grafting in 50 cases of symptomatic nonunion, Shah \& Jones [10] encountered eight cases of fibrous union and elected to stabilize them with a compression screw. These eventually healed without bone grafting. Other authors have suggested that selected non-unions may heal with rigid fixation alone, [5,9-
11], Kim and Lee [12] recorded union with slight resorption at the fracture site in 12 patients with delayed union of the waist of the scaphoid treated by percutaneous screw fixation.

We believe that valuable and reliable information regarding the size of the gap can be gained from plain radiographs. However, CT is needed pre-operatively for its accurate assessment, exclusion of pseudarthroses with sclerosed edges, to assess carpal alignment gap size and sclerosis. We routinely performed CT post-operatively to confirm union, as recommended by many authors $[\mathbf{1 3 , 1 4}]$.

There are no clearly reported data on the extent of bone resorption at the nonunion site and its effect on the need for bone grafting, nor on the method of measurement of gap and sclerosis except in single report in literature [15], in this study we used this method mentioned before in the literature part to assess the gap size and sclerosis on $1 \mathrm{~mm}$ cuts CT films. But still this method has its defects as the maximum gap may be present in the coronal cuts not only the sagittal so we suggest to measure the gap according to the largest diameter either on sagittal or coronal cuts, also the method of measuring sclerosis didn't define the thickness of sclerosis but only its extent.

In Slade and Dodd's classification grade III is assigned for minimum sclerosis, so how much of sclerosis is defined as minimum, and for grade IV \& V cyst formation and sclerosis how much of sclerosis is amenable for percutaneous grafting and doesn't necessitate open debridement of fracture edges, Also for grade IV \& V aligned fractures with gap $<5 \mathrm{~mm} \&>5 \mathrm{~mm}$ and $<10 \mathrm{~mm}$ respectively they stated that they need bone graft even percutaneously, while according to Mahmoud \& koptan [16] these fractures healed properly with rigid fixation only without the need for bone graft which 
was consistent with our results in the study as we had 17 patients grade IV of non-union and 14 grade $\mathrm{V}$ and both groups healed with rigid fixation only without the need for bone graft.

Moreover on analyzing the data we have we found no relation between gap size and delay of time to union, the determinant factor was the time to operation as the more the time since injury, the longer the time the fracture needed to heal.

We recommend this method of treatment for patients who are middle-aged, without previous wrist surgery, who have a nonunion of a fracture of the waist of the scaphoid with no deformity and without avascular necrosis or ligamentous injury, and who have had a CT scan to exclude excessive sclerosis and confirm adequate bone for proximal and distal purchase of the threads of the screw. We also recommend post-operative immobilization for six weeks and restriction of activities until union. Percutaneous fixation of scaphoid although is not technically demanding yet it has a learning curve until mastering the technique.Selected nonunited undisplaced fractures of the scaphoid, even those with a gap > $2 \mathrm{~mm}$, have the biological ability but not the mechanical stability for bone healing. If this is provided by percutaneous rigid fixation, these fractures will unite without requiring a bone graft.

\section{References}

1- DUPPE H., JOHNELL O., LUNDBORG G., KARLSSON M. and REDLUND-JOHNELL I.: Long-term results of fracture of the scaphoid: A follow-up study of more than thirty years. JBJS, 76A: 249-252, 1994.

2- WOLFE S.W.: Fractures of the Carpus: Scaphoid Fractures. IN: Berger R.A., Weiss A.P. Hand surgery. Philadelphia, Lippincott Williams \& Wilkins, USA, 21: 397-402, 2004.

3- CAPO J.T., ORILLAZA N.S. and SLADE J.F.: Percutaneous Management of Scaphoid Non-unions. Tech. Hand. Surg., 13: 23-29, 2009.
4- BULLENS P.H., DRIESPRONG M., LACROIX M. and VEGTER J.: Treatment of Scaphoid Non-Union with a Percutaneous Cortico-cancellous Bone Graft. JHS [Br]., 30: 365,2005

5- WOZASEK G.E. and MOSER K.: Percutaneous screw fixation for fractures of the scaphoid. JBJS Br., 73: 13842, 1991.

6- HADDAD F.S. and GODDARD N.J.: Acute percutaneous scaphoid fracture fixation: A pilot study. JBJS Br., 80-B: 95-9, 1998.

7- BARTON N.J.: Apparent and partial non-union of the scaphoid. JHS Br., 21: 496-500, 1996.

8- JONES NF, BROWN EE, MOSTOFI A, et al.: Healing of a Scaphoid Nonunion Using Human Bone Morphogenetic Protein, JHS., 30 (3): 528-33, 2005.

9- COSIO M.Q. and CAMP R.A.: Percutaneous pinning of symptomatic scaphoid non-unions. JHS Am., 11: 350-5, 1986.

10- SHAH J. and JONES W.A.: Factors affecting the outcome in 50 cases of scaphoid nonunion treated with Herbert screw fixation. JHS Br., 73-B: 680-5, 1991.

11- TARAS J.S., SWEET S., SHUM W., WEISS L.E., BARTOLOZZI A.: Percutaneous and arthroscopic screw fixation of scaphoid fractures in the athlete. Hand. Clin., 15: 467-73, 1999

12- KIM J.K., KIM J.O. and LEE S.Y.: Volar Percutaneous Screw Fixation for Scaphoid Waist Delayed Union. Clin. Orthop. Relat. Res., 468: 1066-71, 2010.

13- RING D., JUPITER J.B. and HERNDON J.H.: Acute fractures of the scaphoid. J. Am. Acad. Orthop. Surg., 8: 225-31, 2000

14- SLADE $3^{\text {rd }}$ J.F. and GILLON T.: Retrospective review of 234 scaphoid fractures and non-unions treated with arthroscopy for union and complications. Scand. J. Surg., 97: 280-9, 2008.

15- AMIRFEYZ R., BEBBINGTON A., DOWNING N.D., ONI J.A. and DAVIS T.R.D.: Isplaced scaphoid waist fractures: The use of a week 4 CT scan to predict the likelihood of union with nonoperative treatment. JHS Eur. Vol., 36: 498, 2011.

16- MAHMOUD M. and KOPTAN W.: Percutaneous screw fixation without bone grafting for established scaphoid nonunion with substantial bone loss. JBJS Br., 93-B: 9326, 2011. 


\section{تثبيت عدم إلتئام عظمة الزورقية عن طريق تدخل جراحى محدود

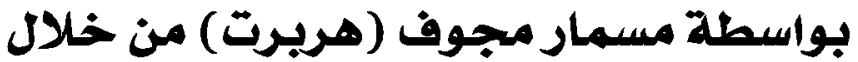

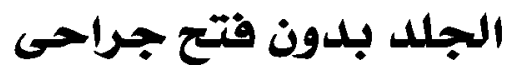

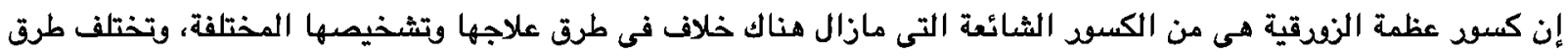

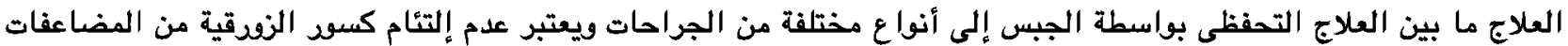

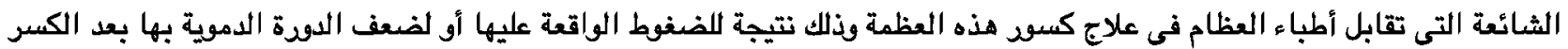

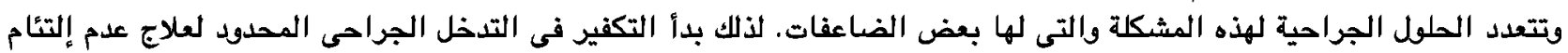
الزندقية ولكن هذا الأسلوب ينطبق على بعض هذه الهذه والكسود وليست كل الحالات.

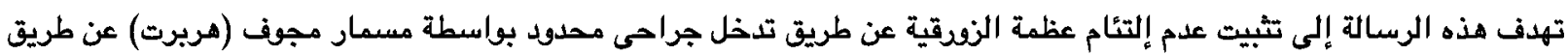

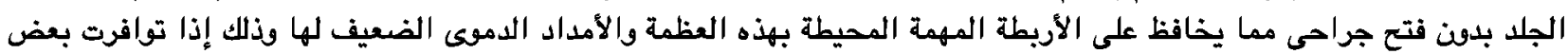

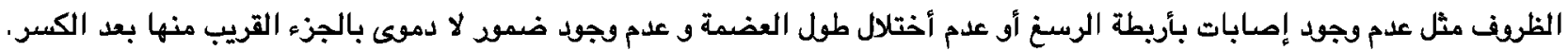

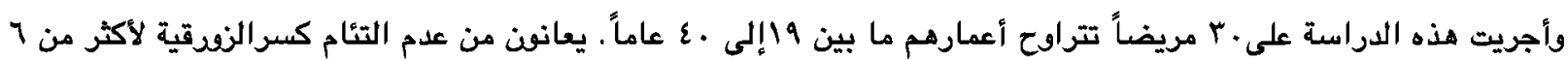

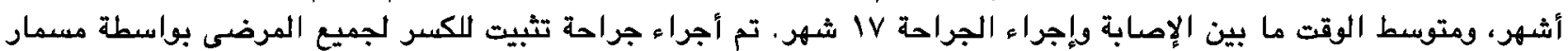
(هربرت) كما تم عمل تثقيب متعدد للتكلس حل الكسر قبل التتبيت. تم متابعة الحالات بواسطة الأشعة السينية والمقطعية لفترة متوسطها

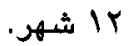

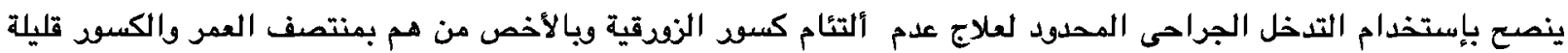

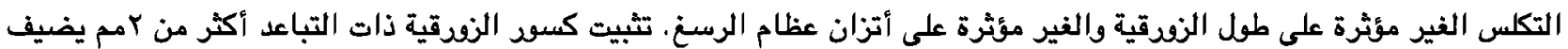

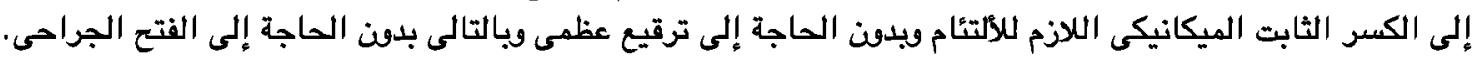

